\title{
THE HOMOTOPY TYPE OF HOMEOMORPHISMS OF 3-MANIFOLDS
}

\section{BY EUGENIA CESAR DE SÁ1 AND COLIN ROURKE}

The purpose of this paper is to announce some results on homeomorphism groups of 3-manifolds. These results together with the Smale conjecture (a proof of which has recently been announced by A. Hatcher) essentially reduce the computation of the homotopy type of the homeomorphism group of a 3-manifold to an analysis of a certain associated configuration space (see \$2) and the homotopy types of the homeomorphism groups of the prime factors. For a prime 3-manifold, this homotopy type is known (a) if the manifold is $P^{2}$-irreducible and sufficiently large [2], (b) for $S^{1} \times S^{2}$ and $S^{1} \underset{\sim}{2} S^{2}$ (see §3).

The results announced here are an extension of the first part of the first author's Ph.D. thesis and use the methods of this thesis. None of our results depends on the Smale conjecture but we have indicated where the Smale conjecture simplifies the conclusion.

1. Preliminaries. Let $M$ be a compact 3-manifold (possibly with boundary) and let $H(M)$ denote the group of PL homeomorphisms of $M$ fixed on $\partial M$. This group has the same homotopy type as the (topological) homeomorphism group of $M$ (fixed on $\partial M$ ) and also, assuming the Smale conjecture, as the diffeomorphism group of $M$.

Let $H(M, D) \subset H(M)$ denote the subgroup of homeomorphisms which are in addition fixed on a standard 3-disc $D$ in int $M$. We remind you that there is a fibration:

$$
H(M, D) \subset H(M) \rightarrow E(D, M)
$$

where $E(D, M)$ denotes the space of PL embeddings of $D$ in int $M . E(D, M)$ has the homotopy type

$$
\begin{array}{ll}
M \times P L_{3} & \text { if } M \text { is orientable, } \\
M \underset{\sim}{\times} P L_{3} & \text { if } M \text { is nonorientable, }
\end{array}
$$

where the twisted product is defined by interchanging the components of $P L_{3}$ around orientation reversing loops in $M$.

Received by the editors June 6, 1978.

AMS (MOS) subject classifications (1970). Primary 57A10, 57E05; Secondary 57E20, 57D15, 57D 50, 58D05.

${ }^{1}$ Supported by the Institut Nacional de Investigacão Cientifico, Portugal. 
We also remind you that $P L_{3}=H\left(\mathbf{R}^{3}, 0\right)$ and that the Smale conjecture is equivalent to the homotopy equivalence $\mathrm{O}_{3} \simeq \mathrm{PL}_{3}$.

The automorphism groups of $M$ and $(M, D)$ are defined by

$$
\text { Aut } M=\pi_{0}(H(M)), \quad \operatorname{Aut}(M, D)=\pi_{0}(H(M, D)) \text {. }
$$

2. The main theorem. Let

$$
M=P_{1} \# \cdots \# P_{n} \#, S^{1} \underset{(\sim)}{\times} S^{2}
$$

be a decomposition of $M$ into prime factors, where the $P_{i}$ are irreducible and $\#_{j} S^{1} \times(\sim) S^{2}$ denotes the connected sum of $j$ copies of $S^{1} \times S^{2}$ or the twisted $S^{2}$-bundle $S^{1} \underset{\sim}{\sim} S^{2}$ over $S^{1}$.

THEOREM 1. There is a homotopy equivalence

$$
\left.H(M, D) \simeq \underset{i=1}{\times} H\left(P_{i}, D\right) \times \underset{j}{\times} \Omega P L_{3}\right) \times \Omega C .
$$

Here $\mathrm{X}_{j} \Omega P L_{3}$ denotes the product of $j$ copies of the loop space on $P L_{3}$ and $\Omega C$ is the loop space on an associated configuration space $C$ defined roughly as follows: a point of $C$ corresponds to a presentation of $M$ as a connected sum or to a presentation of a 3-manifold with the same prime factors as $M$. More precisely, each factor of $M$ is supposed parallelised (or the orientation double cover is parallelised for the non-orientable factors); this allows us to speak of standard discs of either orientation at any point of $M$. We start with $S^{3}=D_{-}^{3} \cup D_{+}^{3}$ and choose a number of points $p_{1}, \ldots, p_{t}, t \leqslant n$, and unordered pairs of points $q_{1}, r_{1}, \ldots, q_{s}, r_{s}, s \leqslant j$, all distinct lying in int $D_{+}^{3}$. Let $D=D_{-}^{3}$. At each point standard disjoint discs are chosen and a selection of prime factors $P_{\boldsymbol{i}_{1}}, \ldots$, $\boldsymbol{P}_{\boldsymbol{t}_{t}}$ are glued in at $\boldsymbol{p}_{1}, \ldots, p_{t}$ using the chosen discs. The discs corresponding to $q_{i}, r_{i}$ are cut out and the boundaries glued together to form $s$ handles, which may be twisted. In the resulting manifold more points $p_{t+1}, \ldots, p_{u}, u \leqslant n$, and pairs $q_{s+1}, r_{s+1}, \ldots, q_{v}, r_{v}, v \leqslant j$, are chosen and the process is repeated using factors of $M$ not used at the first stage. The process stops when all the factors of $M$ have been used. This set of choices is a point of $C$ and identifications are made corresponding to making the choices in a different order and to the possibility of two or more of the $P_{i}$ being the same and so on. A point of $C$ thus gives a 3-manifold which may be homeomorphic to $M$ (i.e. if all the orientations were correct in the case when $M$ is orientable). The identifications can be summarised by saying that two points are identified if the resulting connected sum decompositions are the same (in other words the same or isomorphic factors are glued in the same places). The base point of $C$ is determined by the given decomposition of $M$.

Information on $\pi_{1}(C)=\pi_{0}(\Omega C)$ will be given in $\$ 5$. 
3. The case $M=S^{1} \times_{(\sim)} S^{2}$. In this case $C$ has the homotopy type of the real projective plane $P^{2}$ and we deduce:

Corollary 1.

$$
H\left(S^{1} \underset{(\sim)}{\times} S^{2}, D\right) \simeq \Omega P^{2} \times \Omega P L_{3} .
$$

We can also determine the homotopy type of $H\left(S^{1} \times(\sim) S^{2}\right)$ :

THEOREM 2. (a) $H\left(S^{1} \times S^{2}\right) \simeq H\left(S^{1}\right) \times Q$ where $Q \subset H\left(S^{1} \times S^{2}\right)$ is the space of homeomorphisms that send the base point to the 2-sphere over the base point and respect the orientation on $S^{1}$, and there is a fibration

$$
\Omega \mathrm{PL}_{3} \rightarrow Q \rightarrow P L_{3}
$$

which splits if the Smale conjecture is true.

(b) There are fibrations:

$$
\widetilde{Q} \rightarrow\left(S^{1} \underset{\sim}{x} S^{2}\right) \rightarrow H\left(S^{1}\right), \quad \Omega P L_{3} \rightarrow \widetilde{Q} \rightarrow P L_{3}
$$

where $\widetilde{Q} \subset H\left(S^{1} \times S^{2}\right)$ is the subspace of the homeomorphisms which send the base point to the 2-sphere over the base point and respect the orientation on $S^{1}$.

4. The case where the factors $P_{i}$ are $P^{2}$-irreducible sufficiently large. Using Hatcher [2] and the fibration in $\$ 1$ we can deduce that

$$
\pi_{n} H\left(P_{i}, D\right) \cong \pi_{n+1} P L_{3}, \quad n \geqslant 1,
$$

and that

$$
0 \rightarrow \mathrm{Z}_{2} \rightarrow \pi_{0} H\left(P_{i}, D\right) \rightarrow \text { Aut }^{+} \pi_{1} P_{i} \rightarrow 0
$$

is exact, where Aut ${ }^{+} \pi_{1} M$ is the group of automorphisms which respect the orientation homomorphism. For $P_{i}$ orientable Aut ${ }^{+} \pi_{1} P_{i} \cong \pi_{0} S H\left(P_{i}, x_{0}\right)$ [2] where $S H\left(P_{i}, x_{0}\right)$ is the subgroup of the orientation preserving homeomorphisms fixed on a point $x_{0}$ in int $P_{t}$.

Corollary 2. If $M=\#_{j} S^{1} \times_{(\sim)} S^{2} \#_{i} P_{i}$ where the $P_{i}$ are $P^{2}$-irreducible sufficiently large then

$$
\pi_{n} H(M, D) \cong \pi_{n+1} C \oplus\left(\bigoplus_{i+j} \pi_{n+1} P L_{3}\right), \quad n \geqslant 1 .
$$

5. Application to the automorphism group. We now describe certain specific automorphisms of $M$.

(1) Permutations. Choose two factors $P_{i}$ and $P_{i}$ which are homeomorphic. Slide these factors inside $D_{+}^{3}$ without meeting the other factors to interchange them. This corresponds to a loop in $C$ which interchanges the corresponding points $p_{i}, p_{i}$. There are similar "permutations" using two handles or two ends of the same handle.

(2) SLIDES. Choose a factor $P_{i}$, say, and a loop in $M$ with $P_{i}$ and $D$ removed. Slide $P_{i}$ around this loop. This corresponds to the loop in $C$ in which 
$p_{i}$ is taken around the chosen loop. There are similar "slides" using one end of a handle.

TheOREM 3. The image of $\pi_{0}(\Omega C)$ in $\operatorname{Aut}(M, D)$ is generated by slides and permutations and hence every automorphism (orientation preserving if $M$ is orientable) of a 3-manifold is a composition of slides, permutations and automorphisms of the factors.

Now consider the homeomorphism $\operatorname{Aut}(M, D) \stackrel{\pi}{\rightarrow}$ Aut $\pi_{1} M$ that associates to a homeomorphism the induced automorphism on $\pi_{1}$. It is possible to describe explicitly the automorphisms of $\pi_{1} M$ corresponding to slides and permutations. In fact, Aut $\pi_{1} M$ is generated by the images of slides, permutations and by the automorphisms of the fundamental groups of the factors and it follows from Theorem 3 and [1] that

COROLlary 3. There is an injection

$$
\pi_{0}(\Omega C) \rightarrow \text { Aut } \pi_{1} M
$$

Combining Corollary 3 with Waldhausen's results (see [2]) we can deduce:

Corollary 4. If $M=\#_{i} P_{i} \#_{j} S^{1} \times_{(\sim)} S^{2}$ where the $P_{i}$ are $P^{2}$-irreducible sufficiently large, then there is an exact sequence

$$
0 \rightarrow \bigoplus_{i+j} \mathrm{Z}_{2} \rightarrow \operatorname{Aut}(M, D) \stackrel{\pi}{\rightarrow} G \rightarrow 0
$$

where $G$ is a subgroup of Aut $\pi_{1} M$ which can be described explicitly.

Each $\mathbf{Z}_{2}$-factor in the kernel of $\pi$ comes either from a rotation parallel to the separating sphere of an irreducible factor or from a rotation parallel to the belt-sphere of a handle (see also [3] and [4]).

\section{REFERENCES}

1. D. I. Fuchs and D. J. Rabinovitch, On the automorphism group of free products. I, II, Math. Sb. 50 (1940), 265-276; 9 (1941), 183-220.

2. A. Hatcher, Homeomorphisms of sufficiently large $P^{2}$-irreducible 3-manifolds, Topology 15 (1976), 343-348.

3. H. Hendriks, Applications de la théorie d'obstruction en dimension 3, C. R. Acad. Sci. Paris, Sér. A 276 (1973), $1101-1104$.

4. F. Laudenbach, Topologie de la dimension trois: homotopie et isotopie, Astérisque 12, Soc. Math de France.

DEPARTMENT OF MATHEMATICS, UNIVERSIDADE DO PORTO, PORTO, PORTUGAL (Current address of Eugénia César de Sá)

FACULTY OF MATHEMATICS, THE OPEN UNIVERSITY, WALTON HALL, MILTON KEYNES MK7 6AA, ENGLAND

Current address (Colin Rourke): UNIVERSITY OF WARWICK, MATHEMATICS INSTITUTE, COVENTRY, CV4 7AL, ENGLAND 